\title{
Sexually transmitted infections, the silent partner in HIV-infected women in Zimbabwe
}

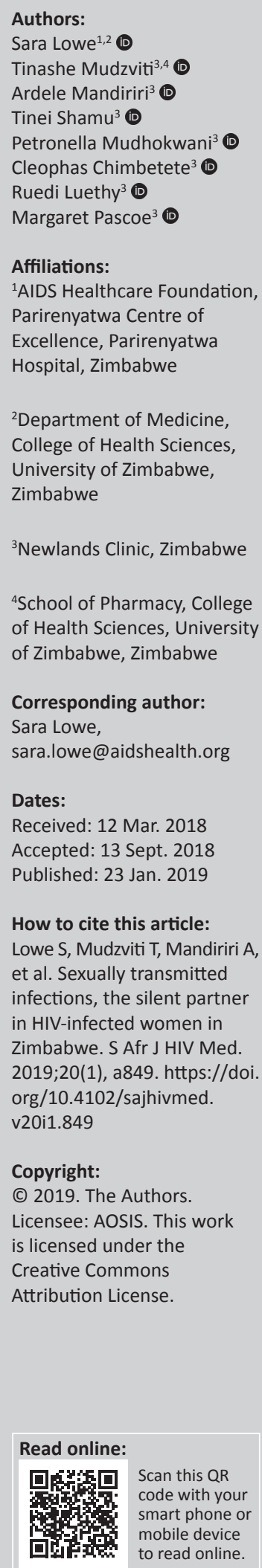

Background: Coinfection rates of HIV and sexually transmitted infections (STIs) are not widely reported in Zimbabwe and no local guidelines regarding the screening of STIs in people living with HIV exist.

Objectives: This cross-sectional study was conducted to determine the prevalence and associated risk factors for STI coinfection in a cohort of HIV-infected women.

Methods: Between January and June 2016, 385 HIV-infected women presenting for routine cervical cancer screening were tested for five STIs: Neisseria gonorrhoeae (NG), Chlamydia trachomatis (CT), Trichomonas vaginalis (TV), Herpes Simplex Virus (HSV) type 2 and Treponema pallidum (TP). Socio-demographic characteristics and sexual history were recorded. Multiple logistic regression was used to identify factors associated with the diagnosis of non-viral STIs.

Results: Two hundred and thirty-three participants (60.5\%) had a confirmed positive result for at least one STI: HSV 2 prevalence 52.5\%, TV 8.1\%, CT 2.1\%, NG 1.8\% and TP $11.4 \%$. Eightyseven per cent of the women were asymptomatic for any STI; $62.3 \%$ of women with a non-viral STI were asymptomatic. Women who had attended tertiary education were $90 \%$ less likely to have a non-viral STI (adjusted odds ratio [aOR]: 0.10, 95\% confidence interval [CI]: 0.03-0.39, $p<0.01)$. Having more than three lifetime sexual partners was a significant predictor for a nonviral STI diagnosis (aOR: 3.3, 95\% CI: 1.5-7.2, $p<0.01$ ).

Conclusion: A high prevalence of predominantly asymptomatic STIs is reported in a cohort of HIV-infected women. Syndromic management results in underdiagnosis of asymptomatic patients. More than three lifetime sexual partners and less formal education are risk factors for coinfection with non-viral STI. High-risk women should be screened using aetiological methods.

\section{Introduction}

Sexually transmitted infections (STIs) and their many sequelae are among the top five reasons that adults seek healthcare in low-income settings. ${ }^{1}$ The World Health Organization reports that worldwide more than one million STIs are acquired each day and an estimated 340 million curable bacterial STIs per year. Viral infections constitute a large proportion of prevalent STIs with an estimated 536 million people living with Herpes Simplex Virus (HSV) and 291 million women with Human Papilloma Virus (HPV) infection at any point in time, with the numbers in men likely to be similar. ${ }^{2}$ Adverse events associated with untreated STIs are common: neonatal morbidity and mortality; infertility; ectopic pregnancy and increased risk of transmission of the Human Immunodeficiency Virus (HIV) are all important sequelae. These under recognised STI epidemics constitute a significant global public health threat and have a profound impact on quality of life, particularly among people aged $15-49$ years. ${ }^{3}$

HIV and STIs share a complex bidirectional relationship. STIs increase HIV viral shedding in the genital tract, resulting in significant increases in HIV transmission risk. Local inflammation activates HIV replication in the genital compartment independent of HIV in peripheral blood. An individual may have an undetectable plasma HIV viral load (VL), while the genital tract VL is elevated. ${ }^{4}$ It has been shown that the presence of both ulcerative and non-ulcerative STIs significantly increases the risk of both acquiring and transmitting HIV. ${ }^{5}$ In systematic reviews conducted by Fleming and Wasserheit ${ }^{6}$ and Kalichman et al., ${ }^{7}$ both groups concluded that HIV transmission was facilitated by the presence of other STIs, whether symptomatic or asymptomatic, and that early STI diagnosis and treatment should be part of a high-quality, comprehensive HIV prevention strategy. ${ }^{6,7}, 8$ 
The prevalence of STIs varies according to region, gender and risk group. A number of key populations with high prevalence of STIs have been reported. These include sex workers, their clients and other partners; men who have sex with men; transgender people; people who inject drugs; and people living with HIV (PLWH). Enhanced STI screening is recommended in these key populations. ${ }^{9,10,11}$ However, access and uptake of STI services among these groups is often challenging. In resource-limited settings, there are limited data regarding the prevalence of STIs, with the exception of HIV, which is often the only STI for which functioning surveillance systems are in place. In Zimbabwe, the prevalence of HIV among adults aged 15-64 years is $14.6 \%$, corresponding to approximately 1.2 million PLWH. ${ }^{12}$ Coinfection rates of HIV and STIs are not widely reported and there are no local guidelines regarding the screening for STIs in PLWH. This study was conducted to determine the prevalence of STI coinfection in a cohort of HIV-infected women and to identify associated risk factors for an STI diagnosis.

\section{Methods}

This analytic cross-sectional study was conducted in HIVinfected adult women at Newlands Clinic (NC), Harare, Zimbabwe. NC provides comprehensive HIV care and treatment services to approximately 6000 individuals in the greater Harare urban area. The clinic operates in a publicprivate partnership with the Ministry of Health and Child Care, Zimbabwe. Funding for the clinic is provided by the Ruedi Luethy Foundation and other partners. ${ }^{13}$ Sexually active, non-pregnant adult women ( $\geq 18$ years of age) attending NC for routine annual cervical screening were invited to participate in the study.

\section{Study procedures}

A questionnaire was verbally administered by a trained study nurse, which collected sociodemographic, medical, gynaecological and sexual history data. Current CD4+ count, HIV VL and antiretroviral therapy (ART) history were documented in the medical history. Current CD4+ count and VL were defined as results which had been obtained within the preceding month. The sexual history included questions regarding age of sexual debut, number of sexual partners, type of sexual activity, STI symptoms, previous STI diagnoses, condom use, family planning, sexual orientation and past history of sexual abuse.

On completion of the questionnaire, a complete abdominal and gynaecological examination including the collection of endocervical swabs was conducted. Findings were recorded on a participant's respective case report forms. On completion of endocervical swab collection, the nurse proceeded with an examination of the cervix using the visual inspection with acetic acid and cervicography (VIAC) methodology. The swabs were used for Chlamydia trachomatis (CT), Neisseria gonorrhoeae (NG), and Trichomonas vaginalis (TV) using the Cepheid Xpert® CT/NG and Xpert ${ }^{\circledR}$ TV assays.
A $4 \mathrm{~mL}$ blood sample was collected into a clot activating tube and processed for onsite testing. Processing involved centrifuging of the sample after clotting and harvesting the serum for subsequent tests. Herpes simplex virus type 2 infection was tested in serum using PreCheck HSV 2 IgG test kits. The seroprevalence of syphilis was defined as having a positive treponemal-specific antibody test using the SD Bioline Rapid Antibody Test with or without a positive nontreponemal RPR carbon assay. All participants with confirmed STI diagnoses were managed using an aetiological approach, and respective antibiotic treatment was administered as recommended in the national guidelines. ${ }^{14}$

VL measurements were performed on EDTA plasma using the Roche COBAS Ampliprep and TaqMan version 2.0, while CD4+ counts were measured in whole blood using a Partec Cyflow Counter II.

\section{Statistical analysis}

Data were entered into a Microsoft Access 2016 database and then exported to Microsoft Excel for cleaning. Cleaned data were exported to Stata 12.1 for analysis. Medians and interquartile ranges (IQR) were used to describe continuous data. A maximum $p$-value of 0.05 was considered statistically significant. Unadjusted odds ratios with $95.0 \%$ confidence intervals (CIs) were calculated for risk factors of STIs. Significant risk factors in univariate analysis were further analysed in a multivariable logistic regression to calculate adjusted odds ratios.

\section{Ethical consideration}

The study was approved by the NC Research Unit and the Medical Research Council of Zimbabwe (approval number MRCZ/A/1980). All participants provided written informed consent before enrolling in the study.

\section{Results \\ Participant enrolment}

Between 01 January and 30 June 2016, 385 women were enrolled in the study, 356 (93.0\%) being on ART. The median age of the participants was 41 years (IQR: 35-47). A total of $171(44.0 \%)$ participants were married and 103 (27.0\%) were widows; $86(22.0 \%)$ had seven years or less of education and $57(15.0 \%)$ had reached tertiary education. Table 1 shows the characteristics of the participants in the study.

\section{HIV treatment history}

Of the $356(93.0 \%)$ participants who were taking ART, 324 $(91.0 \%)$ were virologically suppressed with VLs of $<50$ copies / $\mathrm{mL}$. Twelve $(3.0 \%)$ were severely immunocompromised $(\mathrm{CD} 4+$ cell count $<100$ cells $/ \mu \mathrm{L})$, but the majority were immunocompetent with the median CD4+ cell count being 503 (IQR: 347-655) cells/ $\mu \mathrm{L}$. Among those receiving ART, the median duration on ART was 6.2 years (IQR: 3.2-9.0) and 323 women $(84.0 \%)$ were taking a first-line ART regimen. 
TABLE 1: Participant characteristics $(N=385)$

\begin{tabular}{|c|c|c|c|}
\hline \multirow[t]{2}{*}{ Characteristic } & \multicolumn{3}{|c|}{ Frequency } \\
\hline & $n$ & $\%$ & IQR \\
\hline Median age, years & 41 & - & $35-47$ \\
\hline \multicolumn{4}{|l|}{ Marital status } \\
\hline Married & 171 & 44.4 & - \\
\hline Widowed & 103 & 26.8 & - \\
\hline Divorced/separated & 62 & 16.1 & - \\
\hline Single & 49 & 12.7 & - \\
\hline CD4+ cell count (cells $/ \mu \mathrm{L}$ ), median (IQR) & 503 & - & $347-655$ \\
\hline \multicolumn{4}{|l|}{ Viral load } \\
\hline$<1000$ copies $/ \mathrm{mL}$ & 347 & 97.5 & \\
\hline$<50$ copies $/ \mathrm{mL}$ & 324 & 91.0 & \\
\hline Duration on ART, years, median (IQR) & 6.2 & - & $3.2-9.0$ \\
\hline \multicolumn{4}{|l|}{ Sexual history } \\
\hline Age at sexual debut, years, median (IQR) & 19 & - & $17-21$ \\
\hline Condoms use for last sex & 242 & 62.9 & \\
\hline \multicolumn{4}{|l|}{ HIV status of most recent sexual partner } \\
\hline Positive & 196 & 50.0 & - \\
\hline Negative & 66 & 17.1 & - \\
\hline Unknown & 123 & 32.0 & - \\
\hline Documented previous STI diagnosis & 166 & 43.1 & - \\
\hline Reported symptoms of STI & 47 & 12.2 & - \\
\hline Reported sexual abuse & 26 & 6.8 & - \\
\hline Reported domestic violence & 32 & 8.3 & - \\
\hline
\end{tabular}

IQR, interquartile range; ART, antiretroviral therapy; STI, sexually transmitted infections.

\section{Sexually transmitted infection and sexual history}

Forty-three percent of the women reported having a previous STI, diagnosed using the syndromic management approach. The majority of patients $(n=367,95.0 \%)$ reported having had one or no sex partner in the last six months, while $206(54.0 \%)$ had at least three lifetime partners. All participants reported previous engagement in vaginal sex, while $30(8.0 \%)$ and four $(1.0 \%)$ also reported oral and anal sex, respectively. Three of the four who engaged in anal sex reported having used condoms. All of the women were heterosexual.

\section{Sexually transmitted infection prevalence}

Of the 385 participants screened, 233 (61.0\%) women had at least one confirmed result for an STI (HSV-2, TV, NG, CT, syphilis). Seventy-nine (21.0\%) women had at least one non-viral STI (TV, NG, CT, syphilis). Eleven $(3.0 \%)$ women were coinfected with two non-viral STIs: six were positive for syphilis and TV, three for TV and NG and two for TV and CT. Figure 1 shows the percentage of participants diagnosed with each STI. Seropositive HSV 2 prevalence was 52.5\%, while TV, CT and NG were $8.1 \%, 2.1 \%$ and $1.8 \%$, respectively. Syphilis was newly diagnosed in $44(11 \%)$ women, of whom $26(7.0 \%)$ were RPR and syphilis antibody test positive, while 18 were positive for syphilis antibody test only. Among the 79 patients with non-viral STIs, $54(68.0 \%)$ did not have signs or symptoms. Among these 54, 31 (57.0\%) were positive for syphilis, $6(11.0 \%)$ for CT, 4 (7.0\%) for NG and $19(35.0 \%)$ for TV.

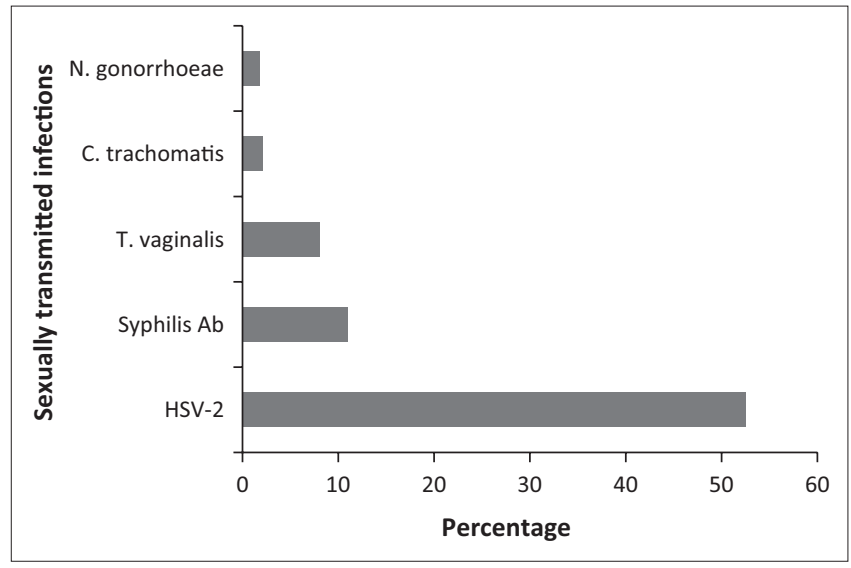

HSV, Herpes simplex virus; $A B$, antibody.

FIGURE 1: Prevalence of sexually transmitted infections $(n=365)$.

\section{Factors associated with a positive non-viral sexually transmitted infection diagnosis}

Women who had attended tertiary education were $90 \%$ less likely to have a non-viral STI compared with those without any education (adjusted odds ratio [aOR]: 0.10, 95\% CI: $0.03-$ $0.39, p<0.01$ ). Those with $\geq 3$ lifetime partners were 3.5 times more likely (aOR: $3.49,95 \%$ CI: $1.64-7.40, p<0.01$ ) to have a non-viral STI compared with those with only one. Women with STI signs and symptoms were three times more likely (aOR: 2.89, 95\% CI: 1.54-5.40, $p<0.01$ ) to have a non-viral STI than those with no signs and symptoms. Table 2 shows the association of selected risk factors with an STI diagnosis.

\section{Discussion}

Our study reports a high prevalence of STIs in a cohort of HIVinfected women in Zimbabwe. Sixty-one per cent of women had a positive confirmatory test for any STI and approximately one in five women was diagnosed with a treatable non-viral STI. The majority of the women in this study (62.0\%) were asymptomatic at the time of diagnosis of non-viral STIs and would therefore not have received treatment using the current syndromic management guidelines. A wide variation in STI prevalence data collected mostly through antenatal programmes has been presented across different low- and middle-income countries..$^{15}$ In a similar study conducted in Khartoum, Sudan, only $17.7 \%$ of the attending antenatal clinics were found to harbour at least one non-viral STI (TV, NG, CT, TP). All the participants in the Khartoum study were HIV-negative. ${ }^{16}$ Untreated symptomatic or asymptomatic STIs are not only associated with significant morbidity, but are also associated with an increased risk of transmission of HIV. ${ }^{8}$ The presence of both ulcerative and non-ulcerative STIs has been associated with increased concentrations of HIV RNA in mucosal secretions, plasma and decreasing CD4+ cell counts. ${ }^{7}$ Identifying these hidden infections with thorough sexual history taking, genital examination and aetiological diagnosis has both individual and public health benefits.

Reported syphilis prevalence in this study is significantly higher $(11.0 \%)$ than what has been previously described in 
TABLE 2: Patient characteristics as risk factors for non-viral sexually transmitted infections.

\begin{tabular}{|c|c|c|c|c|c|c|}
\hline \multirow[t]{2}{*}{ Risk factor } & \multicolumn{2}{|c|}{$\begin{array}{l}\text { All women } \\
N=385\end{array}$} & \multicolumn{2}{|c|}{$\begin{array}{c}\text { Non-viral STI positive } \\
n=79\end{array}$} & \multirow[t]{2}{*}{ OR $(95 \% \mathrm{Cl}, p)$} & \multirow[t]{2}{*}{ aOR $(95 \% \mathrm{Cl}, p)$} \\
\hline & $n$ & $\%$ & $n$ & $\%$ & & \\
\hline \multicolumn{7}{|l|}{ Age (years) } \\
\hline$\leq 25$ & 20 & 5.2 & 7 & 35.0 & - & - \\
\hline $26-35$ & 80 & 21.9 & 22 & 27.5 & $0.51(0.25-1.99,0.51)$ & - \\
\hline$>35$ & 285 & 78.1 & 50 & 17.5 & $0.39(0.09-1.04,0.06)$ & - \\
\hline \multicolumn{7}{|l|}{ Education } \\
\hline Primary & 62 & 16.1 & 19 & 30.7 & $0.73(0.27-1.98,0.54)$ & $0.79(0.28-2.24,0.65)$ \\
\hline Secondary & 242 & 62.9 & 47 & 19.4 & $0.40(0.17-0.97,0.04)$ & $0.39(0.15-1.00,0.05)$ \\
\hline Tertiary & 57 & 14.8 & 4 & 7.0 & $0.13(0.03-0.47,<0.01)$ & $0.10(0.03-0.39,<0.01)$ \\
\hline \multicolumn{7}{|c|}{ Lifetime partners } \\
\hline 1 & 91 & 23.6 & 9 & 9.9 & - & - \\
\hline 2 & 88 & 22.9 & 13 & 14.8 & $1.57(0.64-3.90,0.32)$ & $1.55(0.60-3.99,0.37)$ \\
\hline$\geq 3$ & 206 & 53.5 & 57 & 27.7 & $3.49(1.64-7.40,<0.01)$ & $3.27(1.49-7.19,<0.01)$ \\
\hline Casual & 12 & 3.1 & 0 & - & - & - \\
\hline Regular & 373 & 96.9 & 79 & 21.2 & - & - \\
\hline \multicolumn{7}{|c|}{ Condom use at last sexual intercourse } \\
\hline Yes & 246 & 63.9 & 53 & 21.5 & - & - \\
\hline No & 139 & 36.1 & 26 & 18.7 & $0.83(0.50-1.41,0.51)$ & - \\
\hline \multicolumn{7}{|c|}{ STI signs and symptoms } \\
\hline No & 312 & 81.0 & 54 & 17.3 & - & \\
\hline Yes & 73 & 19.0 & 25 & 34.3 & $2.49(1.41-4.38,<0.01)$ & $2.89(1.54-5.40,<0.01)$ \\
\hline \multicolumn{7}{|c|}{ Previous STI } \\
\hline No & 219 & 56.9 & 39 & 17.8 & - & - \\
\hline Yes & 166 & 43.1 & 40 & 24.1 & $1.46(0.89-2.40,0.13)$ & - \\
\hline \multicolumn{7}{|c|}{ Age at sexual debut (years) } \\
\hline$<16$ & 25 & 6.5 & 7 & 28.0 & - & - \\
\hline $16-20$ & 237 & 61.6 & 59 & 24.9 & $0.85(0.34-2.14,0.73)$ & $1.30(0.49-3.51,0.60)$ \\
\hline$>20$ & 123 & 31.9 & 13 & 10.6 & $0.39(0.16-0.93,0.03)$ & $0.60(0.19-1.86,0.37)$ \\
\hline Yes & 52 & 13.5 & 11 & 13.9 & $1.05(0.51-2.14,0.90)$ & - \\
\hline
\end{tabular}

$\mathrm{OR}$, odds ratio; $\mathrm{Cl}$, confidence interval; $\mathrm{STI}$, sexually transmitted infection.

Note: Bold values indicate statistically significant, $p$-value $<0.05$

Zimbabwean cohorts. Gwanzura et al. reported a prevalence of $2.3 \%$ in male factory workers. The prevalence of active syphilis in HIV-infected women and all antenatal clinic attendees has been previously reported at $4.0 \%$ and $1.2 \%$, respectively. ${ }^{17,18,19}$ This increase is in keeping with global trends of syphilis incidence and higher prevalence in PLWH. ${ }^{7,20}$ Rekart et al. suggest a novel hypothesis that ART may potentially alter both innate and acquired immune responses in ways that may lead to enhanced susceptibility to syphilis. ${ }^{21}$ With a larger proportion of patients now taking ART (93.0\% in this study), this enhanced susceptibility could be a significant factor contributing to the rise in incident syphilis in HIV-infected treated adults. The high prevalence of syphilis in our study provides further evidence to support the need to enhance the surveillance of syphilis and provide effective syphilis control programmes within existing HIV care and treatment programmes.

The second most prevalent non-viral STI in this study was TV, which was confirmed in $8.0 \%$ of the participants. Similar to other STIs, it is increasingly recognised that TV plays an important role in increasing the risk of both acquisition and onward transmission of HIV. Using mathematical models, Quinlivan et al. estimated that 22.0\% of projected HIV transmissions from women in the
United States are attributable to TV infections and up to $2.0 \%$ of all HIV transmissions in the United States may be related to TV infection. ${ }^{22}$

The prevalence of infection with chlamydia varies significantly between regions, countries and risk groups. The prevalence of NG and CT in our study was $1.8 \%$ and $2.1 \%$, respectively. Studies conducted in Kenya, Zimbabwe, Nigeria and South Africa have shown that the prevalence of CT varied between $6.0 \%$ and $20.0 \%$ in these countries. ${ }^{23,24,25} \mathrm{~A}$ Zimbabwean study investigating the prevalence of NG and $\mathrm{CT}$ in 5448 men and women congregating at bottle stores revealed a baseline prevalence of $2.2 \%$ and $3.8 \%$, respectively. The HIV status of these individuals was, however, not recorded. The factors associated with incident infection were being female and having more than one sexual partner. The proportion of patients with incident infection not reporting symptoms was greater than $75.0 \%$ for both infections; ${ }^{26,27}$ this is consistent with other studies in PLWH in the region.

An HSV 2 seropositivity rate of $52.0 \%$ is in keeping with global published rates in PLWH of $50.0 \%-90.0 \% .{ }^{27}$ Data from a review conducted by Smith and Robinson showed that up to $90.0 \%$ of PLWH in some settings were coinfected with HSV-2. ${ }^{27}$ Herpes Simplex Virus type 2and HIV are 
synergistic co-pathogens. The parallel intersecting epidemics of the HIV and HSV infections are well documented, and studies indicate that HSV 2 plays an important role in the spread of HIV and affects virological control of the coinfected untreated patient. ${ }^{28}$

Analysis from this study suggests that women with less formal education were more likely to be diagnosed with an STI; this is consistent with findings from other similar studies. Quinlivan et al. found a five-fold increase in risk of infection with TV in women living with HIV with low reported education status. ${ }^{22}$ Suggested explanations for this commonly reported observation are that lesser educated women are less likely to be employed and therefore are more dependent on their sexual partners or more likely to engage in transactional sex. Negotiating safe sex in both these situations may be more challenging. . $^{29,30}$

Multivariate analysis revealed that women reporting more than three lifetime sexual partners were three times as likely to be diagnosed with a non-viral STI. Taking a thorough sexual history is an important tool in risk profiling of patients, and in countries where resources are limited, it can enable healthcare professionals to target high-risk patients for targeted screening. Participants in this study were not recruited based on symptoms. However, on completion of a detailed sexual history, $13.0 \%$ of women reported ongoing symptoms consistent with an STI diagnosis. Possible reasons for participants delaying in seeking treatment include stigma associated with an STI diagnosis. ${ }^{31}$ The level of education attained by an individual may also have a bearing on whether they will seek treatment as they may not be in a position to relate ongoing symptoms to a possible STI diagnosis. This is of importance in countries like Zimbabwe where STI treatment programmes focus on syndromic management approach.

The prevalence of previous or current sexual abuse and/or intimate partner violence (IPV) was reported in this study. Our results are consistent with published data reporting a strong association between IPV and HIV infection. ${ }^{32}$ Studies from Tanzania and Rwanda report that women experiencing IPV are up to three times more likely to acquire HIV. ${ }^{33,34}$ Studies also report an association between sexual abuse in childhood and increased sexual risk behaviours in adulthood leading to higher rates of STIs, including HIV. ${ }^{35}$ Importantly, many of the women in the study had not previously disclosed this sensitive information despite long-term and recurrent attendance at a health facility, highlighting the need for confidential routine sexual history taking in all HIV-infected women.

This study highlights an unrecognised burden of STIs and defines an at-risk population of HIV-infected women. An important limitation of the study is, however, the lack of generalisability of our results to all HIV-infected women, as participants were recruited from one urban site. The cohort also did not include key populations such as sex workers or adolescents and young adults. The investigators did not survey any additional anatomical sites; consequently, the results for this study focus on vaginal and cervical STIs (except for syphilis). Women were asked to report historical events which may introduce recall bias particularly relating to number of sexual partners and condom use.

In conclusion, we have reported a high prevalence of nonviral STIs in a cohort of largely asymptomatic HIV-infected women. Those reporting more than three lifetime partners and with less formal education were at higher risk of STI acquisition. The major strategy in most resource-poor settings for STI control is focused on syndromic management of genitourinary infections. Although we recognise this is an important public health measure, this hidden epidemic may be associated with significant morbidity and drives onward HIV transmission. STI control remains an important aspect of HIV prevention. These results emphasise the need for identification of high-risk women through routine sexual history checking and targeted aetiological screening of highrisk individuals for asymptomatic STIs. There is an increased role for diagnostic technology which can be used for surveillance and STI screening of at-risk women. Polymerase chain reaction and point-of-care diagnostic technology need to be included as part of rapid STI treatment modalities.

\section{Acknowledgements Competing interests}

The authors declare that they have no financial or personal relationships that may have inappropriately influenced them in writing this article.

\section{Authors' contributions}

S.L. was responsible for the conception of the study and its design, drafting of the manuscript and analysis of data. P.M. was responsible for data collection. M.P. supervised the research process and contributed to the analysis of the data and write-up. A.M. was responsible for managing the study data, follow-up of patients and assisted with the write-up. T.M., T.S., R.L. and C.C. assisted with study conception, data analysis and write-up. All the authors revised the draft critically and gave final approval of the version to be published. All authors read and approved the final manuscript.

\section{References}

1. Dallabetta G, Field ML, Lage M, Islam QM. STDs: Global burden and challenges for control. In: Dallabetta G, Laga M, Lamptey P, editors. Control of sexually transmitted diseases: A handbook for the design and management of programs. Durham, NC: Family Health International/The AIDS Control and Prevention Project(AIDSCAR), 2006; p. 23-52.

2. World Health Organization. Sexually transmitted infections. Fact sheet no. 110 2016. Available from: http://apps.who.int/iris/bitstream/handle/10665/82207/ who_rhr_13.02_eng.pdf

3. Newman L, Rowley J, Vander Hoorn S, et al. Global estimates of the prevalence and incidence of four curable sexually transmitted infections in 2012 based on systematic review and global reporting. PLoS One. 2015;10(12):e0143304. https://doi.org/10.1371/journal.pone.0143304

4. Kalichman SC, Cherry C, White D, Jones M, Kalichman M. The Achilles' heel of HIV treatment for prevention: History of sexually transmitted coinfections among people living with HIV/AIDS receiving antiretroviral therapies. I Int Assoc Physicians AIDS Care (Chic). 2011;10(6):365-372. https://doi.org/10.1177/ Physicians AIDS Ca
1545109711418120 
5. Johnson LF, Lewis DA. The effect of genital tract infections on HIV-1 shedding in the genital tract: A systematic review and meta-analysis. Sex Transm Dis. 2008;35(11):946-959. https://doi.org/10.1097/OLQ.0b013e3181812d15

6. Fleming DT, Wasserheit JN. From epidemiological synergy to public health policy and practice: The contribution of other sexually transmitted diseases to sexua transmission of HIV infection. Sex Transm Infect. 1999;75(1):3-17. https://doi. org/10.1136/sti.75.1.3

7. Kalichman SC, Pellowski J, Turner C. Prevalence of sexually transmitted coinfections in people living with HIV/AIDS: Systematic review with implication for using HIV treatments for prevention. Sex Transm Infect. 2011;87(3):183-190. https://doi.org/10.1136/sti.2010.047514

8. Gray RH, Wawer MJ, Brookmeyer R, et al. Probability of HIV-1 transmission pe coital act in monogamous, heterosexual, HIV-1-discordant couples in Rakai, Uganda. Lancet. 2001;357(9263):1149-1153. https://doi.org/10.1016/S01406736(00)04331-2

9. World Health Organization. Prevention and treatment of HIV and other sexually transmitted infections for sex workers in low- and middle income countries: Recommendations for a public health approach [homepage on the Internet] 2012 [cited $2013 \mathrm{Jul}$ 21]. Available from: http://www.who.int/hiv/pub/guidelines/ sex worker/en

10. World Health Organization. Prevention and treatment of HIV and other sexually transmitted infections for men who have sex with men and transgender people: Recommendations for a public health approach [homepage on the Internet]. 2012 [cited $2013 \mathrm{Jul} 21$ ]. Available from: http://www.who.int/hiv/pub/guidelines/ msm guidelines 2011/en

11. World Health Organization, United Nations Office on Drugs and Crime, UNAIDS Technical guide for countries to set targets for universal access to HIV prevention, treatment and care for injecting drug users [homepage on the Internet]. 201 revision [cited 2013 Jul 21]. Available from: http://www.who.int/hiv/pub/idu/ targets_universal_access/en/index.html

12. Hakim A, Radin E, Ruangtragool L, et al. Correlates of being outside the 90-90-90 cascade among adults ages 15-64 years in Zimbabwe. 9th IAS Conference on HIV Science (IAS 2017); 23-26 July 2017; Paris, France; 2017.

13. Shamu T, Chimbetete C, Shawarira-Bote S, Mudzviti T, Luthy R. Outcomes of an HIV cohort after a decade of comprehensive care at Newlands Clinic in Harare Zimbabwe: TENART cohort. PLoS One. 2017;12(10):e0186726. https://doi. org/10.1371/journal.pone.0186726

14. Ministry of Health and Child Care Z. 7th essential medicines list and standard treatment guidelines for Zimbabwe. The National Medicine and Therapeutics Polic Advisory Committee (NMTPAC) Ministry of Health and Childcare; 2015. p. 88-100.

15. Joseph Davey DL, Shull HI, Billings JD, Wang D, Adachi K, Klausner JD. Prevalence of curable sexually transmitted infections in pregnant women in low- and middleincome countries from 2010 to 2015: A systematic review. Sex Transm Dis. income countries from 2010 to 2015: A systematic review. Sex Tran
2016;43(7):450-458. https://doi.org/10.1097/OLQ.0000000000000460

16. Abdelrahim NA, Ahmed HI, Fadl-Elmula IM, Bayoumi MA, Homeida MM Sexually transmitted infections other than HIV/AIDS among women of low socioeconomic class attending antenatal clinics in Khartoum, Sudan. Int J STD AIDS. 2017;28(8):781-787. https://doi.org/10.1177/0956462416668080

17. Gwanzura L, Latif A, Bassett M, Machekano R, Katzenstein DA, Mason PR Syphilis serology and HIV infection in Harare, Zimbabwe. Sex Transm Infect. 1999;75(6):426-430. https://doi.org/10.1136/sti.75.6.426

18. Celum C, Wald A, Hughes J, et al. Effect of aciclovir on HIV-1 acquisition in herpes simplex virus 2 seropositive women and men who have sex with men: $A$ randomised, double-blind, placebo-controlled trial. Lancet. 2008;371(9630):21092119. https://doi.org/10.1016/S0140-6736(08)60920-4

19. Kurewa NE, Mapingure MP, Munjoma MW, Chirenje MZ, Rusakaniko S, StrayPedersen $\mathrm{B}$. The burden and risk factors of sexually transmitted infections and reproductive tract infections among pregnant women in Zimbabwe. BMC Infect Dis. 2010;10:127. https://doi.org/10.1186/1471-2334-10-127
20. Patton ME, Su JR, Nelson R, Weinstock $H$, Centers for disease $C$, prevention Primary and secondary syphilis - United States, 2005-2013. MMWR Morb Mortal Wkly Rep. 2014;63(18):402-406

21. Rekart ML, Ndifon W, Brunham RC, et al. A double-edged sword: Does highly active antiretroviral therapy contribute to syphilis incidence by impairing immunity to Treponema pallidum? Sex Transm Infect. 2017:93(5):374-378. https://doi. to Treponema pallidum? Sex Transm

22. Quinlivan EB, Patel SN, Grodensky CA, Golin CE, Tien HC, Hobbs MM. Modeling the impact of Trichomonas vaginalis infection on HIV transmission in HIV-infected individuals in medical care. Sex Transm Dis. 2012:39(9):671-677. https://doi. org/10.1097/OLQ.0b013e3182593839

23. Adachi K, Klausner JD, Bristow CC, et al. Chlamydia and Gonorrhea in HIV-infected pregnant women and infant HIV transmission. Sex Transm Dis. 2015;42(10): 554-565. https://doi.org/10.1097/OLQ.0000000000000340

24. Morhason-Bello I, Ojengbede O, Oladokun A, et al. The prevalence and outcome of asymptomatic chlamydial infection screening among infertile women attending gynecological clinic in Ibadan, South West Nigeria. Ann Med Health Sci Res. 2014;4(2):253-257. https://doi.org/10.4103/2141-9248.129057

25. Takuva S, Mugurungi O, Mutsvangwa J, et al. Etiology and antimicrobial susceptibility of pathogens responsible for urethral discharge among men in Harare, Zimbabwe. Sex Transm Dis. 2014;41(12):713-717. https://doi.org/10.1097/ OLQ.0000000000000204

26. Detels R, Green AM, Klausner JD, et al. The incidence and correlates of symptomatic and asymptomatic Chlamydia trachomatis and Neisseria gonorrhoeae infections in selected populations in five countries. Sex Transm Dis. 2011:38(6):503-509. https://doi.org/10.1097/OLQ.0b013e318206c288

27. Smith JS, Robinson NJ. Age-specific prevalence of infection with herpes simplex virus types 2 and 1: A global review. J Infect Dis. 2002;186 (Suppl 1):S3-28. https://doi.org/10.1086/343739

28. Hill C, McKinney E, Lowndes CM, et al. Epidemiology of herpes simplex virus types 2 and 1 amongst men who have sex with men attending sexual health clinics in England and Wales: Implications for HIV prevention and management. Euro Surveill. 2009;14(47):19418. https://doi.org/10.2807/ese.14.47.19418-en

29. Kakaire O, Byamugisha JK, Tumwesigye NM, Gamzell-Danielsson K. Prevalence and factors associated with sexually transmitted infections among HIV positive women opting for intrauterine contraception. PLoS One. 2015;10(4):e0122400. https://doi.org/10.1371/journal.pone.0122400

30. Muula AS. HIV infection and AIDS among young women in South Africa. Croat Med J. 2008;49(3):423-435. https://doi.org/10.3325/cmj.2008.3.423

31. Crabbe F, Carsauw H, Buve A, Laga M, Tchupo JP, Trebucq A. Why do men with urethritis in Cameroon prefer to seek care in the informal health sector? Genitourin Med. 1996;72(3):220-222.

32. Jewkes RK, Levin JB, Penn-Kekana LA. Gender inequalities, intimate partner violence and HIV preventive practices: Findings of a South African cross-sectional study. Soc Sci Med. 2003;56(1):125-134. https://doi.org/10.1016/S02779536(02)00012-6

33. Maman S, Mbwambo JK, Hogan NM, et al. HIV-positive women report more lifetime partner violence: Findings from a voluntary counseling and testing clinic in Dar es Salaam, Tanzania. Am J Public Health. 2002;92(8):1331-1337. https:// doi.org/10.2105/AJPH.92.8.1331

34. Dunkle KL, Jewkes RK, Brown HC, Gray GE, McIntryre JA, Harlow SD. Genderbased violence, relationship power, and risk of HIV infection in women attending antenatal clinics in South Africa. Lancet. 2004;363(9419):1415-1421. https://doi. org/10.2105/AJPH.92.8.1331

35. Cohen M, Deamant C, Barkan S, et al. Domestic violence and childhood sexual abuse in HIV-infected women and women at risk for HIV. Am J Public Health 2000;90(4):560-565. https://doi.org/10.2105/AJPH.90.4.560 